\title{
INNOVATION AND KNOWLEDGE MANAGEMENT IN PRECISION FARMING
}

\author{
Natalia Bakhtadze ${ }^{1}$, Evgeny Maximov ${ }^{1}$, Danila Donchan ${ }^{1}$, Lamara Kozlovskaya $^{2}$ \\ and Natalia Maximova ${ }^{1}$ \\ ${ }^{I}$ V.A.Trapeznikov Institute of Control Science, 65, Profsoyuznaya street, Moscow, Russia \\ ${ }^{2}$ Russian Timiryazev State Agrarian University, Russia
}

\begin{abstract}
This paper is devoted to the development of information systems for precision farming, which make it possible to use fertilizer and fuel more efficiently on the basis of information technologies and intelligent predictive models, which reduces the cost of production and increases the efficiency of agricultural production. In addition, a long-term agronomic and ecological effect can be achieved due to more careful tillage and reduced use of nitrogen fertilizers.

Description of the creating a knowledge base and models of grain yield depending on the mode of mineral fertilizer application based on intelligent associative search algorithms describes.
\end{abstract}

\section{KEYWORDS}

Precision Farming, Soft Sensors, Predictive Models, Knowledge Management

\section{INTRODUCTION}

Currently, in different countries, integrated systems for the differential application of nitrogen fertilizers are used quite widely (Jordan et al., 2004; Aubert et al., 2012). However, these systems are costly and provide no means all the possible information about the dynamics of the properties of the field pieces, affecting the growth of productivity and determining the application rate. No less costly and time consuming is an approach based on extracting data from multiple sensors, with subsequent processing using specialized software (Ehlert et al., 2009).

Recently, new information technologies and intellectual methods of analysis have become more and more popular (Pavon-Pulido et al., 2017; Biomax, 2018; IBM, 2018). An alternative could be the development of information systems and so-called soft sensors - virtual analyzer to build a predictive model of indicators for the fields of land, based on the intelligent analysis of already available measurement of current and historical data and knowledge base Bakhtadze et al., 2007, 2012, 2015).

Knowledge refers to the laws that are extracted from data analysis, refined as information accumulates. On the basis of predictive models, not a specific control algorithm should be implemented, but a certain strategy, within which not only parameters can change, but also the structure of the system. This approach is significantly less costly and technologically efficient. To build the model, a temporary database of archival and current technological information is formed. After determining the forecast for the output based on the current state of the object, this virtual base is destroyed without memorization. The algorithm does not adjust the approximating model of the real process - it builds a new model for each point in time, and the parameter estimates at any time point are the best in terms of the minimum of the root-mean-square error.

The associative search algorithm is a procedure based on data mining, in particular, clustering methods are used. On the basis of these methods, a set of inputs (in the general case, multidimensional) close to the current input vector in the sense of a certain criterion is selected from the data archive at the time studied. The criterion is called the associative pulse and can be both a functional and a logical or fuzzy function. Further, on the basis of the classical (non-recurrent) least squares method (OLS), the output value is determined at the next time point. Under the assumption that input actions satisfy Gaussian-Markov conditions, the estimates obtained by the least squares method are consistent, unbiased statistically effective. 
The advantage of this approach is that it allows you to build locally linear models for objects characterized by the most different types of non-linearities. The construction of virtual predictive models by the method of associative search is an automated procedure for generating a control action based on the image of the situation being analyzed. The algorithms have demonstrated highly accurate results for non-linear non-stationary objects in industry and energy. The use of such algorithms in Agromanagement systems allow to synthesize the effective management solutions in precise (coordinate) farming systems.

Thus, the article presents methods for creating high-performance innovative systems for supporting the management of differential fertilizer application based on data mining. The proposed methods have no analogues in the world scientific literature, and, at the same time, are due to practical significance.

\section{CREATING A KNOWLEDGE BASE, AND MODEL BUILDING}

The yield forecast, for example, of wheat for a specific field is based on the analysis of the dynamics of several indicators. We analyzed both natural factors and indicators defined farming mode.

The following factors were selected for the study and construction of the forecast model:

- Soil-climatic zone.

- Climate characteristic for the last 10 years: monthly precipitation, average daily air temperature monthly.

it is planned to take into account: the moisture reserve in the soil layer is up to $1 \mathrm{~m}$ at the critical phases of grain development: sowing - seedlings, tillering-booting, earing - pouring

- Field history for the last 7-10 years:

- Preceding and currently cultivated crops

- Crop yield (it is planned to take into account also the quality of grain)

- Amount (doses) of the applied mineral fertilizers for the previous crop and for the cultivated crop, including the frequency and doses of nitrogen fertilizers.

It is also planned to take into account in the model for further research:

- Soil characteristic of the field, including: soil difference, humus content, texture, and pH acidity (for sod-podzolic and gray forest soils),

- The field relief, including the steepness of the slopes, the exposure of the slopes, the closure of the relief elements,

- Provision of soil with nutrients: phosphorus, potassium (in the arable layer) - once every 5 years or after harvesting the previous culture and nitrogen - every spring, before applying nitrogen fertilizers at the beginning of the growing season in the upper soil layer (up to $60 \mathrm{~cm}$ ).

Figure 1 presents the database structure. The experimental database contains information from 30 agricultural enterprises.

Further the Base of inductive knowledge was developed. Under the knowledge in the system refers to patterns that are identified on the basis of data analysis of the real functioning of the object, the model of which is built. Patterns can be formalized by a set of "inputs" and corresponding to these sets of "outputs" of the model.

Further, using the statistical analysis, and application of the associative search algorithms, the analysis was made on the influence of various factors yields of main crops. The main purpose of the analysis was to assess whether the impact of the mode of application of mineral fertilizers on crop yields.

To provide the analysis process with the necessary data from the knowledge base, a software module was developed that samples data using SQL queries and then performs the necessary software processing so that the data can be correctly processed by associative search algorithms. 


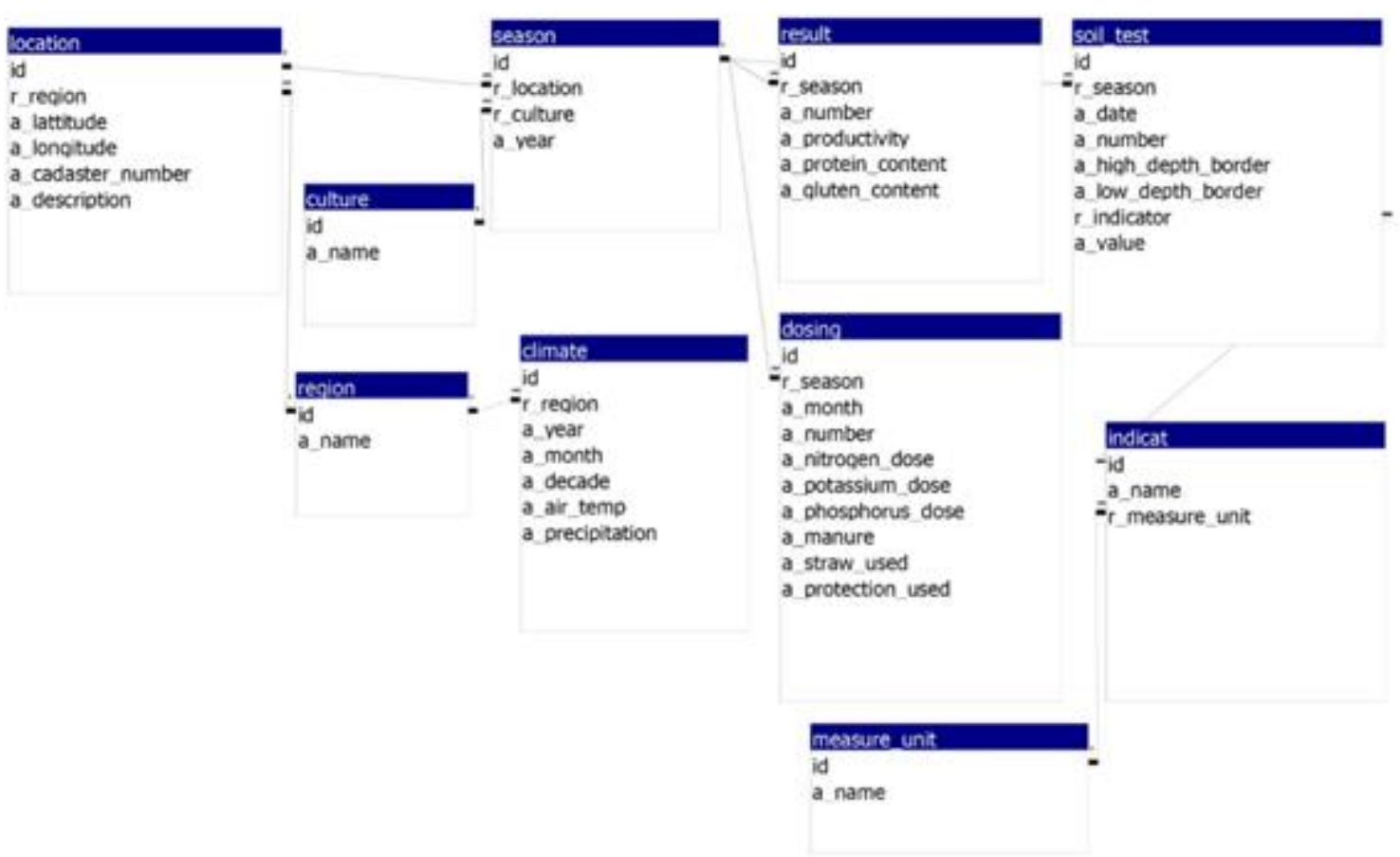

Figure 1. Database Structure

\section{ASSOCIATIVE SEARCH TECHNIQUE}

Algorithms based on knowledge revealed from process history (inductive knowledge, persistently enriched) implement an intelligent approach to constructing identification models. The intelligence is applying knowledge (Knowledge Based) revealed from historical data on the basis of their analysis (Data Mining).

The process of knowledge processing in the intelligent system is reduced to recovering (associative search of) knowledge over its fragment. Meanwhile, the knowledge may be interpreted as associative connections between images. As an image, we will use "sets of indicators", that is components of input vectors, input variables.

The criterion of closeness between images may be formulated in very different manners. In the most general case, it may be a logic function, the predicate. In a particular case, when sets of indicators are vectors in $n$-dimensional space, the criterion of closeness may be a distance in this space.

The associative search process may be implemented either as a process of recovering the image over partially given indicators (or recovering a knowledge fragment under the conditions of incomplete information; as a rule, just this process is simulated in different models of the associative memory), or as a process of searching another images that are associatively connected with the given one, respecting to other time instants.

In (Bakhtadze et al., 2007) an approach to form the support of decision making on the control is proposed, based on dynamic modeling the associative search procedure. Results of adoption of the associative search algorithms developed by the authors for industrial processes of the chemical and petroleum manufacturing, processes of control in intelligent power networks (smart grids), trading processes, transport logistic processes.

The method of the associative search consists in constructing virtual predicting models. The method assumes constructing predicting model of a dynamic plant, being new under each $t$, by use of a set of history data ("associations") formed at the stage of learning and adaptively corrected in accordance to certain criteria, rather than approximating real process in the time. Within the present context, linear dynamic model is of the form: 


$$
y_{N}=\sum_{i=1}^{m} a_{i} y_{N-i}+\sum_{j=1}^{r_{S}} \sum_{S=1}^{S} b_{j, s} x_{N-j, s}, \quad \forall j=\overline{1, N}
$$

where: $y_{N}$ is the prediction of the plant output at the time instant $N, x_{N}$ is the vector of input actions, $m$ is the memory depth in the output, $r_{S}$ is the memory depth in the input, $S$ is the dimension of the input vectors, $a_{i}$ and $b_{j, s}$ are the tuned coefficient, meanwhile $x_{N-j, s}$ are selected disregarding the order of the chronological decreasing, have been referred as the associative pulse.

Let us note that this model is not classical regression one: there are selected certain inputs in accordance to a certain criterion, rather than all chronological "tail". The algorithm of deriving the virtual model consists in constructing at each time instant an approximating hypersurface of the space of input vectors and single-dimensional outputs. To construct a virtual model, corresponding to a certain time instant, from the archive, there are selected input vectors being in a certain sense close to the current input vector. An example of selecting the vectors is described below. The dimension of this hyper-surface is selected heuristically. Again, by use of classical (non-recursive) least squares (LS) method there is determined the output value (modeled signal) in the next time instant. Meanwhile, each point of the global non-linear surface of the regression is formed in the result of using linear "local" models, in each new time instant.

Unlikely to classical regression models, for each fixed time instant from the process history, there are selected input vectors being close to the current input vector in the sense of a certain criterion (rather than the chronological sequence as it is done in regression models). Thus, in equation (1) $r_{s}$ is the number of vectors from the archive (from the time instant 1 to the time instant $N$ ), selected in accordance to the associative search criterion. At each time segment $[N-1, N]$ there is selected a certain set of $r_{s}$ vectors, $1 \leq r_{s} \leq N$. The criterion of selecting the input vectors from the archive to derive the virtual model in the given time instant over the current plant state may be as follows.

Let us introduce as a distance (a norm in $\mathfrak{R}^{S}$ ) between points of the $S$-dimensional space of inputs the value:

$$
d_{N, N-j}=\sum_{S=1}^{S}\left|x_{N, S}-x_{N-j, S}\right|, \forall j=\overline{1, N},
$$

where $x_{N, S}$ are components of the input vector at the current time instant $N$. By virtue of a property of the norm («the triangle inequality», we have:

$$
d_{N, N-j} \leq \sum_{s=1}^{S}\left|x_{N, S}\right|+\sum_{s=1}^{S}\left|x_{N-j, s}\right|, \quad \forall j=\overline{1, N},
$$

To derive an approximating hypersurface for the vector $x_{N}$ let us select from the archive of the input data such vectors $x_{N-j}, j=\overline{1, N}$ that for a set $D_{N}$ the condition will hold:

$d_{N, N-j} \leq d_{N}+\sum_{s=1}^{S}\left|x_{N-j, S}\right| \leq D_{N}, \forall j=\overline{1, N}$, where $\quad \sum_{S=1}^{S}\left|x_{N, S}\right|=d_{N}$.

where $D_{N}$ may be selected, for instance, from the condition:

$$
D_{N} \geq 2 d_{N}^{\max }=2 \max _{j} \sum_{S=1}^{S}\left|x_{N-j, s}\right| .
$$

Under the assumptions that the input actions meet the Gauss-Markov conditions, the estimates obtained via the LS method are unbiased and statistically effective. 


\section{CASE STUDIES}

The yield of calculated by the model was compared with the real yield for different regions of the Russian Federation.

Model. Experimental fields of the Nizhny Novgorod Research Institute of Agriculture from 2008 to 2016.

When building this model, data were used not only from the Nizhny Novgorod Research Institute of Agriculture, but also from other regions of the middle zone with similar climatic conditions. The following influencing factors were identified: temperature in April - August; precipitation in April; precipitation in August

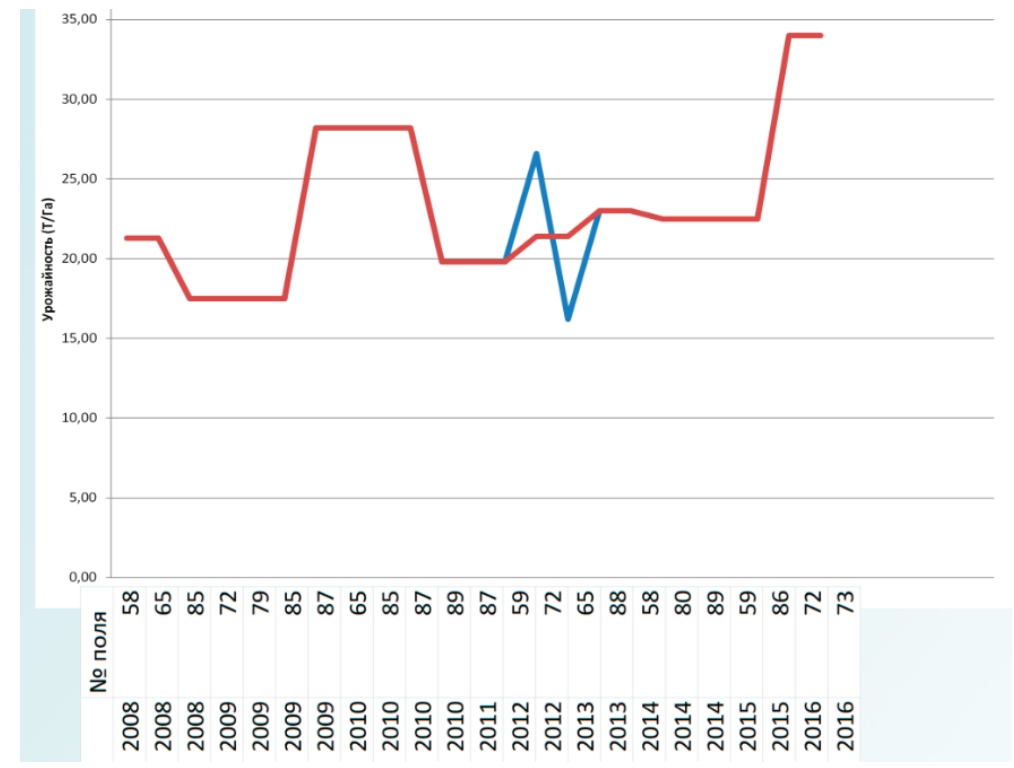

Figure 2. Dynamics of wheat yield - and forecast - using the associative search method in the fields of Nizhny Novgorod Research Institute of Agriculture (Russian Federation)

The accuracy of the model was high. KMK (coefficient of multiple correlation) by the model is 0.95 .

\section{PERSPECTIVE RESEARCH}

Along with the yield forecast, some agrochemical indicators could be useful. For fields with similar characteristics (soil type, water-holding properties) from regions with a similar climate, it is possible to predict the concentration of certain chemical elements and compounds in the soil after applying fertilizers.

The input parameters of the forecast model will be statistical data of previous periods, as well as climatic indicators, under the influence of which chemical processes take place in the soil, contributing to (or hindering) the absorption of fertilizers by plants. In addition to mineral fertilizers, other chemicals also get into the soil - means of protection against pests, etc. They may contain compounds that can affect the values of agrochemical parameters. This factor will also be taken into account in the model. For the cultivation of various crops by agricultural enterprises, technological maps are formed in which, taking into account the climatic zone, the recommended scenarios of the work of agricultural producers are given, which make it possible to achieve a given yield.

For the best assimilation of minerals contained in the soil, different cultures need to moisture and temperature of the air were in certain ranges, beyond which the metabolism slows down. If we trace the dependence of the dynamics of the amount of various trace elements in the soil on the amount of precipitation and temperature (i.e., determine the rate at which plants absorb minerals found in the soil or coming in with fertilizers for different values of climatic indicators), we can determine the required amount 
of fertilizers applied depending on the region's climate (it may be necessary to apply less fertilizer, because the climate does not allow the plants to assimilate all the mineral substances introduced, or on the contrary, plants may need additional feeding during the season). The input parameters of the model will be air temperature and rainfall, and output - agrochemical indicators, which can be used to determine in dynamics the extent to which plants absorb minerals from the soil. Similarly, you can choose the recommended type and number of means of protection, taking into account the indicators of crop rotation, climate, soil characteristics, desired yield. As a result, in essence, technological maps will be developed that more accurately take into account the specifics of a particular field. To determine the need for additional irrigation and its volumes, data on the content of productive moisture in the soil are needed. Based on the dependence of indicators of soil moisture on precipitation, you can make a forecast that allows you to calculate the time and amount of artificial irrigation required. Fields are sampled by culture (possibly also by variety and reproduction), taking into account water permeability and moisture capacity. Then the input parameter will be the amount of precipitation, the output - the content of productive moisture in the soil.

\section{CONCLUSIONS}

The article presents the results on the development of field plots models and methods to support the management of differential fertilization.

The knowledge-based identification and simulation algorithms developed in the framework of this work have demonstrated highly accurate results. The use of such algorithms in Agromanagement systems allow to synthesize the effective management solutions in precise (coordinate) farming systems.

\section{REFERENCES}

Aubert B. A, A. Schroeder, J. Grimaudo, 2012. IT as enabler of sustainable farming: An empirical analysis of farmers' adoption decision of precision agriculture technology. Decision Support Systems, v.54, p.510-520

Bakhtadze, N. and Sakrutina, E., 2015. The Intelligent Identification Technique with Associative Search. International Journal of Mathematical Models and Methods in Applied Sciences 9, pp. 418-431.

Bakhtadze, N., Kulba, V., Lototsky, V. and Maximov, E. Identification-based approach to soft sensors design. Proceedings of IFAC Workshop of Intelligent Manufacturing Systems. Alicante, Spain (2007), pp. 86-92.

Bakhtadze, N., Lototsky, V., Vlasov, S., and Sakrutina, E., 2012. Associative Search and Wavelet Analysis Techniques in System Identification. IFAC Proceedings, Volumes 45(16), pp. 1227-1232.

Biomax. Agriculture IoT System, 2018. Available online: http://www.biomax.com.tw/en/product/ Agriculture-IoTSystem/agriculture_iot_system.html (accessed on 16 April 2018).

Ehlert D., Adamek R., Horn H.J. Vehicle based laser range finding in crops. Sensors, 2009, pp. 3679-3694. doi: 10.3390/s90503679. [PMC free article] [PubMed] [CrossRef].

IBM. IBM Research Precision Agriculture. 2018. Available online: https://www.ibm.com/us-en/ marketplace/agri-resrchinnov (accessed on 16 April 2018).

Jordan C., Shi Z, Bailey J.S., Higgiris A., 2004. Sampling strategies for mapping 'within-field' variability in the dry matter yield and mineral nutrient status of forage grass crops in cool temperature climates. Precision Agriculture 4, pp. 69-86.

Pavon-Pulido N., Lopez-Riquelme J.A., Torres R., Morais R., Pastor J.A., 2017. New trends in precision agriculture: A novel cloud-based system for enabling data storage and agricultural task planning and automation. Precis. Agric. 18, pp.1038-1068. doi: 10.1007/s11119-017-9532-7. [CrossRef]. 